\title{
Competence-based fuzzy skill functions is
}

\author{
Wen Sun ${ }^{\mathrm{a}}$, Jinjin Li ${ }^{\mathrm{b}, *}$, Fucai Lin ${ }^{\mathrm{b}, \mathrm{c}}$, Bo Wang ${ }^{\mathrm{d}}$, Zhaorong $\mathrm{He}^{\mathrm{a}}$ \\ ${ }^{a}$ Department of Mathematics, Shantou University, Shantou 515063, China \\ ${ }^{b}$ School of Mathematics and Statistics, Minnan Normal University, Zhangzhou 363000, China \\ ${ }^{c}$ Fujian Key Laboratory of Granular Computing and Applications, Minnan Normal University, Zhangzhou 363000, \\ China \\ ${ }^{d}$ School of Mathematical Sciences, Huaqiao University, Quanzhou 362021, China
}

\begin{abstract}
Fuzzy skill functions connect knowledge states at the performance level with latent cognitive abilities at the competence level. Given that there may exist precedence relations among skills, the main idea of this study is trying to develop fuzzy competence structures restricted on the fuzzy sets of skills that are likely to occur. We consider the knowledge structures determined by fuzzy skill functions and fuzzy competence structures. More precisely, disjunctive fuzzy skill functions delineate knowledge spaces if the fuzzy competence structures are union-closed, while conjunctive fuzzy skill functions delineate simple closure spaces if the fuzzy competence structures are intersection-closed. In general, competence-based fuzzy skill function delineates knowledge structures depending on the effective competence states. Correspondingly, we build algorithms that delineate knowledge structures via competence-based fuzzy skill functions. In the courses of these algorithms, one does not need to list all fuzzy competence states of fuzzy competence structures.
\end{abstract}

Keywords: Fuzzy competence structure; Fuzzy skill function; Knowledge structure; Problem function

\section{Introduction}

Knowledge structure theory (KST) was first developed by Doignon and Falmagne (1985). Afterwards, Korossy $(1997,1999)$ introduced an extension of KST based on skills and call it competenceperformance approach. The observable behavior is referred to the performance level and can be explained by the competence level. Skill functions and problem functions establish some connections between competence levels and performance levels. During the past two decades, competence-based knowledge structure theory (Cb-KST) fast prospered for personalised learning and knowledge evaluation (Anselmi, Robusto, \& Stefanutti, 2012; Anselmi, Stefanutti, de Chiusole, \& Robusto, 2017; Heller, Steiner, Hockemeyer, \& Albert, 2006; Heller, Anselmi, Stefanutti, \& Robusto, 2017; Spoto, Stefanutti, \& Vidotto, 2016; Stefanutti, \& de Chiusole, 2017; Stefanutti, 2019).

\footnotetext{
This work is supported by the National Natural Science Foundation of China (No. 11871259, 11971287) and the Key Program of the Natural Science Foundation of Fujian Province (No. 2020J02043).

*Corresponding author

Email addresses: 18wsun2@stu.edu.cn (Wen Sun), jinjinli@mnnu.edu.cn (Jinjin Li), linfucai@mnnu.edu.cn (Fucai Lin), wangbo2013@hqu.edu.cn (Bo Wang), 17zrhe@stu.edu.cn (Zhaorong He)
} 
Item response theory (IRT) and cognitive psychology (de la Torre, 2009; Mislevy, 1996; Zhan, Jiao, \& Liao, 2017) come together with the formal framework for representing cognitive theories provided by cognitive diagnostic models (CDM). Heller et al. (2015) built the connections between CDM and KST, and showed that the MS-DINA (multiple strategy deterministic input noisy ANDgate) model (de la Torre, \& Douglas, 2008; de la Torre, 2011) and the CBLIM (competence-based local independence model) are equivalent. At the beginning, CDM and Cb-KST are dichotomous attributes (skills). Next, polytomous attributes were introduced into CDM (Chen, \& de la Torre, 2013; Karelitz, 2004; von Davier, 2008). Recently, the dichotomous skill functions have been extended to fuzzy skill functions (Sun, Li, Ge, \& Lin, 2021). On the other hand, Bartl and Belohlavek (2011), Stefanutti et al. (2020) and Heller (2021) have generalized quasi-ordinal knowledge spaces to polytomous items. Although there is little communication between the two camps, the developments of CDM and KST are surprisingly similar. However, both fuzzy skill functions in Cb-KST and polytomous attributes in CDM involve to neither the precedence relation of skills, nor fuzzy (polytomous) competence structure on the set of skills.

Throughout this paper, for any set $X$, the collection of all subsets of $X$ is written as $\mathcal{P}(X)$, called the power set of $X$. Moroever, we use the symbol $\mathcal{P}^{*}(X)$ to denote the family $\mathcal{P}(X) \backslash\{\varnothing\}$.

Given that there may exist precedence relations among skills, it is reasonable to use a subset of $\mathcal{P}(S)$ to represent the competence structure (Heller, Ünlü, \& Albert, 2013; Korossy, 1997, 1999). It's natural that a competence structure can be extended to a fuzzy competence structure, and that the Cb-KST can be extended to fuzzy competence-based KST. Similar with the polytomous knowledge structure, we proposed the concept of fuzzy competence structure, in which every fuzzy competence state represents an individual's levels of proficiency of skills in ideal conditions.

For any competence structure $(S, \mathcal{C})$, Korossy $(1997,1999)$ defined a special skill multimap $(Q, S, \mu)$ from $Q$ to $\mathcal{P}^{*}(\mathcal{C} \backslash\{\varnothing\})$, called an interpretation function. Correspondingly, for a fuzzy competence structure $\left(S, V_{s}, \mathcal{C}\right)$, we propose the concept of fuzzy interpretation function, which is a special fuzzy skill function. Some results in the present paper shows that the generalization of conjunctive fuzzy interpretation functions inherits the results in Lemma 11.4.2 and Proposition 11.4.3 (2) (Heller, Ünlü, \& Albert, 2013). However, for fuzzy competence spaces, the generalized results in Lemma 11.4.1 and Proposition 11.4.3 (1) (Heller, Ünlü, \& Albert, 2013) of fuzzy interpretation functions are unnecessarily correct, see Proposition 3 and Remark 8 in this paper.

There are two mainly approaches for constructing knowledge structures in KST. The query approach (Cosyn, \& Thiery, 2000; Heller, 2004; Kambouri, Koppen, Villano, \& Falmagne, 1994) is based on entail relation on $Q$ and the judgements of experienced experts. On the other hand, a fuzzy skill function can be built by experienced experts in empirical situation, and the approach for delineating knowledge structures via competence-based fuzzy skill functions is from the perspective of underlying cognitive abilities.

The aim of the present work is not only to study knowledge structures induced by competencebased fuzzy skill functions, but also to design algorithms for constructing knowledge structures via competence-based fuzzy skill functions. Knowledge structures are completely determined by fuzzy competence structures and fuzzy skill functions. For any fuzzy skill function, if there is no restriction on $S$, then the fuzzy competence structure is $\mathcal{F}(S)$. In most case, a fuzzy competence structure is a family $\mathcal{C}$ of subsets of $\mathcal{F}(S)$. In dichotomous $\mathrm{Cb}-\mathrm{KST}$, an algorithm for constructing knowledge structures via (dichotomous) skill functions was introduced by Düntsch and Gediga $(1995,2002)$. Next, a similar method to delineate knowledge structures via competence-based skill functions was introduced by Heller et al. (2013). Given that it is necessary to list all subsets 
of $S$ with their methods, it's hard to do this job when the number of fuzzy competence states of a fuzzy competence structure is large enough. In this paper, we designed an algorithm for constructing knowledge structures via competence-based fuzzy skill functions just depends on the effective competence states of $(Q, S, \mu)$ in $\mathcal{C}$. On the other hand, Heller introduced two special skill functions: disjunctive skill function and conjunctive skill function (Heller, Ünlü, \& Albert, 2013). Some results of this article show that knowledge spaces can be induced by disjunctive fuzzy skill functions when the fuzzy competence structures are $\sqcup$-closed. Moreover, conjunctive fuzzy skill functions delineate simple closure spaces if the fuzzy competence structures are $\sqcap$-closed. By the characteristics of knowledge structures, we also build algorithms that delineate knowledge structures by disjunctive fuzzy skill functions and conjunctive fuzzy skill functions. These algorithms are only concerned with the atoms of fuzzy competence spaces or minimal intersection generation groups of $\sqcap$-closed fuzzy competence structures.

Section 2 outlines some relevant background of KST, Cb-KST and fuzzy set theory. Fuzzy competence structures are discussed in Section 3. We build algorithms that delineate knowledge structures via competence-based fuzzy skill functions in sections 4 . Section 5 introduces fuzzy interpretation functions. Section 6 summarizes the main results of this study.

\section{KST, Cb-KST and fuzzy set theory: an overview}

A subset of $Q$ that an individual is capable of solving is called a knowledge state, where $Q$ is a set of items. Let $\mathcal{K}$ be a family of knowledge states. A pair $(Q, \mathcal{K})$ is said to be a knowledge structure if $\varnothing, Q \in \mathcal{K}$. Sometimes, we write $\mathcal{K}$ instead of $(Q, \mathcal{K})$. A knowledge structure $(Q, \mathcal{K})$ is said to be a knowledge space if $\mathcal{K}$ is union-closed: $K \cup L \in \mathcal{K}$ for any $K, L \in \mathcal{K}$. A knowledge structure $(Q, \mathcal{K})$ is called a simple closure space if $\mathcal{K}$ is intersection-closed: $K \cap L \in \mathcal{K}$ for any $K, L \in \mathcal{K}$.

Let $\mathcal{G}$ be a collection. The collection $\mathcal{G}^{\prime}=\left\{G^{\prime}: G^{\prime}=\bigcup \mathcal{A}\right.$ for some $\left.\mathcal{A} \subseteq \mathcal{G}\right\}$ is called the span of $\mathcal{G}$. Let $\mathcal{K}$ be a knowledge space. A minimal subfamily $\mathcal{B}$ of $\mathcal{K}$ is said to be the basis of $\mathcal{K}$ if $\mathcal{K}$ is the span of $\mathcal{B}$.

A triple $(Q, S, \mu)$ is called a skill function if $\mu$ is a mapping from $Q$ to $\mathcal{P}^{*}\left(\mathcal{P}^{*}(S)\right)$ and the elements in $\mu(q)$ are pairwise incomparable for every $q \in Q$. In this manner, each element in $\mu(q)$ can be considered as a method for solving $q$, called a competency.

Let $\mathcal{C}$ be a subcollection of $\mathcal{P}(S)$. The pair $(S, \mathcal{C})$ is called a competence structure on $S$ if $\varnothing, S \in \mathcal{C}$. Each element in $\mathcal{C}$ is called a competence state, which represents a subset of skills that can occur. Occasionally, we write $\mathcal{C}$ instead of $(S, \mathcal{C})$. A competence structure $(S, \mathcal{C})$ is called a competence space if $\mathcal{C}$ is union-closed.

Let $(S, \mathcal{C})$ be a competence structure and $(Q, S, \mu)$ a skill function. Define a mapping $p_{\mu}: \mathcal{C} \rightarrow$ $\mathcal{P}(Q)$ by

$$
p_{\mu}(T)=\{q \in Q \mid C \subseteq T \text { for some } C \in \mu(q)\} .
$$

The triple $\left(Q, S, p_{\mu}\right)$ is called the problem function of $(Q, S, \mu)$. A problem function $\left(Q, S, p_{\mu}\right)$ is monotonic if $p_{\mu}\left(T_{1}\right) \subseteq p_{\mu}\left(T_{2}\right)$ for any $T_{1} \subseteq T_{2}$, where $T_{1}, T_{2} \in \mathcal{C}$.

Let $(S, \mathcal{C})$ be a competence structure. Define a mapping $\mu: Q \longrightarrow \mathcal{P}^{*}(\mathcal{C} \backslash\{\varnothing\})$ such that elements in each $\mu(q)$ are pairwise incomparable. The triple $(Q, S, \mu)$ is said to be an interpretation function on $(S, \mathcal{C})$.

Remark 1. For any interpretation function, the assumption 'pairwise incomparability' for each $\mu(q)$ is not required in Korossy $(1997,1999)$. In Heller et al. (2013)), an interpretation function is 
called a skill function if $\mu(q)$ is pairwise incomparable for each $q \in Q$. In this paper, the 'pairwise incomparability' for each $\mu(q)$ is required for any interpretation function. Heller et al. (2017) pointed out that competencies assigned to items are unnecessarily competence states in $\mathcal{C}$. The difference between interpretation functions and skill functions is trivial.

Let $(S, \mathcal{C})$ be a competence structure. For any interpretation function $(Q, S, \mu)$, Korossy (1997, $1999)$ defined a representation function $\left(Q, S, p_{\mu}\right)$ from $\mathcal{C}$ to $\mathcal{P}(Q)$ as follows:

$$
p_{\mu}(C)=\{q \in Q \mid C \in \mu(q)\} .
$$

Heller et al. (2013) thought the concept of representation function is similar to the concept of a problem function.

Several maps related to items and skills are listed in Table 1.

Table 1: Several maps related to items and skills

\begin{tabular}{llcc}
\hline & Definition & Incomparability & Competence structure $(S, \mathcal{C})$ \\
\hline Skill map & $Q \longrightarrow \mathcal{P}^{*}(S)$ & No & $\mathcal{C}=\mathcal{P}(S)$ \\
Skill multimap & $Q \longrightarrow \mathcal{P}^{*}\left(\mathcal{P}^{*}(S)\right)$ & No & $\mathcal{C}=\mathcal{P}(S)$ \\
Skill function & $Q \longrightarrow \mathcal{P}^{*}\left(\mathcal{P}^{*}(S)\right)$ & Yes & $\mathcal{C}=\mathcal{P}(S)$ \\
Interpretation function & $Q \longrightarrow \mathcal{P}^{*}(\mathcal{C} \backslash\{\varnothing\})$ & Yes & $\mathcal{C} \subseteq \mathcal{P}(S)$ \\
Problem function & $\mathcal{C} \longrightarrow \mathcal{P}(Q)$ & No & $\mathcal{C} \subseteq \mathcal{P}(S)$ \\
\hline
\end{tabular}

For detailed contents of KST and Cb-KST, refer to (Falmagne, Koppen, Villano, Doignon, \& Johanessen, 1990; Doignon, 1994; Doignon, \& Falmagne, 1999; Falmagne, \& Doignon, 2011; Falmagne, Albert, Doble, Eppstein, \& Hu, 2013).

Let $X, Y$ be two sets and $Y^{X}$ the set of all mapping from $X$ to $Y$. Some operations on $Y^{X}$ are defined as follows (Zadeh, 1965):

(1) For any $f, g \in Y^{X}, f=g$ if $f(x)=g(x)$ for all $x \in X$.

(2) For any $f, g \in Y^{X}, f \subseteq g$ if $f(x) \leq g(x)$ for all $x \in X$.

(3) For any subset $\left\{f_{i}: i \in I\right\} \subseteq Y^{X}$, define $\bigsqcup_{i \in I} f_{i}(x)=\bigvee_{i \in I} f_{i}(x)$, for all $x \in X$. The mapping $\bigsqcup_{i \in I} f_{i}$ is said to be the supremum of $\left\{f_{i}: i \in I\right\}$.

(4) For any subset $\left\{f_{i}: i \in I\right\} \subseteq Y^{X}$, define $\prod_{i \in I} f_{i}(x)=\bigwedge_{i \in I} f_{i}(x)$, for all $x \in X$. The mapping $\prod_{i \in I} f_{i}$ is said to be the infimum of $\left\{f_{i}: i \in I\right\}$.

Let $\mathbf{I}$ denote the unit closed interval $[0,1]$. The set of all fuzzy set on $S$ is denoted by $\mathcal{F}(S)$, i.e., $\mathcal{F}(S)=\{T \mid T: S \rightarrow \mathbf{I}\}$. We use $T=\left\{\frac{T(s)}{s} \mid s \in S\right\} \in \mathcal{F}(S)$ to denote a fuzzy set $T: S \rightarrow \mathbf{I}$. If $T(s)=0$ for some $s \in S$, then $\frac{T(s)}{s}$ is omitted. Sometimes, a pair $(s, v)$ in $S \times \mathbf{I}$ is denoted by $s^{v}$ and $s^{v} \in T$ expresses the fact that $T(s)=v$. For more about fuzzy set theory, refer to (Zadeh, 1965).

Thereafter, $Q, V_{s}$ and $S$ are always nonempty finite sets.

\section{Fuzzy competence structures}

Stefanutti et al. (2020) and Heller (2021) proposed the concepts of polytomous knowledge structure and polytomous knowledge state. Correspondingly, we introduce the concepts of fuzzy competence structure and fuzzy competence state. For each skill $s \in S$, let $V_{s}$ denote a finite non-empty set and $v \in[0,1]$ for any $v \in V_{s}$. Slightly misusing notation, let $S \times V_{s}$ denote the set $\bigcup_{s \in S}\left(\{s\} \times V_{s}\right)$ of all possible skill $\times$ value combinations. 
Definition 1. (Mirsky, 1971) Let $\mathcal{A}=\left\{A_{i} \subseteq E: i \in I\right\}$. A subset $X \subseteq E$ is called a transversal of $\mathcal{A}$ if there exists a bijection $\psi: X \rightarrow I$ such that $x \in A_{\psi(x)}$ for all $x \in X$.

Let $T$ be a transversal of the collection $\left\{\{s\} \times V_{s}: s \in S\right\}$ of subsets of $S \times V_{s}$. The mapping $\psi: T \rightarrow S$ defined by $\psi\left(s^{v}\right)=s$ for all $s^{v} \in T$ forms a bijection satisfying $s^{v} \in\left\{\psi\left(s^{v}\right)\right\} \times V_{\psi\left(s^{v}\right)}=$ $\{s\} \times V_{s}$. Slightly misusing notation, let $V_{s}^{S}$ denote the set of all transversals for the collection $\left\{\{s\} \times V_{s}: s \in S\right\}$.

Definition 2. Let $S$ be a finite nonempty set of skills, and $V_{s}$ for all $s \in S$ be finite nonempty sets. For any $s \in S$, let $V_{s}$ be endowed with the numerical order with a bottom element 0 and a top element 1 . Moreover, let $\mathcal{C} \subseteq V_{s}^{S}$. A triple $\left(S, V_{s}, \mathcal{C}\right)$ is called a fuzzy competence structure on $V_{s}^{S}$ if $S \times\{0\}, S \times\{1\} \in \mathcal{C}$ and $\bigcup \mathcal{C}=S \times V_{s}$. Each element $C \in \mathcal{C}$ is called a fuzzy competence state.

Example 1. Let $S=\left\{s_{1}, s_{2}, s_{3}, s_{4}\right\}, V_{s_{1}}=V_{s_{4}}=\{0,0.6,0.8,1\}, V_{s_{2}}=\{0,0.7,1\}$ and $V_{s_{3}}=$ $\{0,0.9,1\}$. It is trivial to verify that the family $\left\{C_{i}: 1 \leq i \leq 27\right\}$ in Table 2 is a fuzzy competence structure.

Table 2: A fuzzy competence structure $\left(S, V_{s}, \mathcal{C}\right)$

\begin{tabular}{cccccccccc}
\hline & $s_{1}$ & $s_{2}$ & $s_{3}$ & $s_{4}$ & & $s_{1}$ & $s_{2}$ & $s_{3}$ & $s_{4}$ \\
\hline$C_{1}$ & 0 & 0 & 0 & 0 & $C_{15}$ & 0 & 0.7 & 0.9 & 0.8 \\
$C_{2}$ & 0 & 0.7 & 0 & 0 & $C_{16}$ & 0.6 & 0.7 & 0.9 & 0.6 \\
$C_{3}$ & 0.6 & 0 & 0 & 0.6 & $C_{17}$ & 0.6 & 0.7 & 0.9 & 0.8 \\
$C_{4}$ & 0 & 0.7 & 0.9 & 0 & $C_{18}$ & 0.8 & 0.7 & 0.9 & 0.6 \\
$C_{5}$ & 0.8 & 0 & 0.9 & 0 & $C_{19}$ & 0.8 & 0.7 & 0.9 & 0.8 \\
$C_{6}$ & 0 & 0 & 0.9 & 0.8 & $C_{20}$ & 0.8 & 1 & 0.9 & 0.8 \\
$C_{7}$ & 0.6 & 0.7 & 0 & 0.6 & $C_{21}$ & 1 & 0.7 & 0.9 & 1 \\
$C_{8}$ & 0.6 & 0 & 0.9 & 0.8 & $C_{22}$ & 1 & 0.7 & 1 & 0.6 \\
$C_{9}$ & 0.8 & 0.7 & 0.9 & 0 & $C_{23}$ & 1 & 0.7 & 1 & 0.8 \\
$C_{10}$ & 0.8 & 0 & 0.9 & 0.6 & $C_{24}$ & 1 & 1 & 0.9 & 1 \\
$C_{11}$ & 0.8 & 0 & 0.9 & 0.8 & $C_{25}$ & 1 & 1 & 1 & 0.6 \\
$C_{12}$ & 0.8 & 1 & 0 & 0.8 & $C_{26}$ & 1 & 1 & 1 & 0.8 \\
$C_{13}$ & 1 & 0.7 & 1 & 0 & $C_{27}$ & 1 & 1 & 1 & 1 \\
$C_{14}$ & 1 & 0 & 0.9 & 1 & & & & & \\
\hline
\end{tabular}

Sometimes, we say that $\mathcal{C}$ is the fuzzy competence structure if $S$ and $V_{s}$ are specified.

Definition 3. A fuzzy competence structure $\left(S, V_{s}, \mathcal{C}\right)$ is called a fuzzy competence space if $\mathcal{C}$ is $\sqcup$-closed, i.e., if $C, C^{\prime} \in \mathcal{C}$, then $C \sqcup C^{\prime} \in \mathcal{C}$.

Definition 4. (Stefanutti, Anselmi, de Chiusole, \& Spoto, 2020) Let $X, Y$ be two nonempty sets and $\mathcal{F} \subseteq Y^{X}$. The family $\mathcal{F}^{\prime}=\{\sqcup \mathcal{A}: \mathcal{A} \subseteq \mathcal{F}\}$ is called the span of $\mathcal{F}$, denoted by $\mathbb{S}(\mathcal{F})=\mathcal{F}^{\prime}$.

For any fuzzy competence space $\left(S, V_{s}, \mathcal{C}\right)$, there exists a subfamily of $\mathcal{C}$ spanning $\mathcal{C}$. The minimal subfamily spanning $\mathcal{C}$ can fully characterize $\mathcal{C}$ without losing valuable information.

Definition 5. Let $\left(S, V_{s}, \mathcal{C}\right)$ be a fuzzy competence space. A minimal subfamily $\mathcal{B} \subseteq \mathcal{C}$ is said to be the basis of $\mathcal{C}$ if there exists $\mathcal{B}^{\prime} \subseteq \mathcal{B}$ such that $C=\sqcup \mathcal{B}^{\prime}$ for any $C \in \mathcal{C}$. 
Definition 6. Let $\left(S, V_{s}, \mathcal{C}\right)$ be a fuzzy competence space. For any $v \in V_{s} \backslash\{0\}$ and $s \in S$, a fuzzy competence state $C \in \mathcal{C}$ is said to be a $v$-atom at $s^{v}$ if it satisfies the following two conditions:

(i) $s^{v} \in C$;

(ii) for any $C^{\prime} \in \mathcal{C}$ with $C^{\prime} \sqsubseteq C, s^{v} \in C^{\prime}$ implies $C^{\prime}=C$.

For any skill $s \in S$ and $v \in V_{s} \backslash\{0\}$, the set of all the atoms at $s^{v}$ is denoted by $\sigma_{s^{v}}$. Moreover, the set of all the atoms of $s$ is denoted by $\sigma_{s}=\bigcup_{v \in V_{s} \backslash\{0\}} \sigma_{s^{v}}$. Obviously, $\sigma_{s}$ is nonempty for any $s \in S$, since $\bigcup \mathcal{C}=S \times V_{s}$.

Theorem 1. Every fuzzy competence space $\left(S, V_{s}, \mathcal{C}\right)$ has a basis $\mathcal{B}=\bigcup_{s \in S} \sigma_{s}$.

Proof. Note that $V_{s}$ and $S$ are finite, then the basis $\mathcal{B}$ of $\mathcal{C}$ exists. If there exists $C \in \mathcal{B}$ but $C \notin \bigcup_{s \in S} \sigma_{s}$. Then for any $s \in S, C \notin \sigma_{s}$. It follows that $C \notin \sigma_{s^{v}}$ for any $v \in V_{s} \backslash\{0\}$. Let $C(s)=v_{s}$. Then we can choose $C_{s^{v_{s}}} \in \mathcal{C}$ such that $s^{v_{s}} \in C_{s^{v_{s}}} \sqsubset C$. Thus, $C=\sqcup_{s \in S} C_{s^{v_{s}}}$, contradicting $C \in \mathcal{B}$. Hence, $\mathcal{B} \subseteq \bigcup_{s \in S} \sigma_{s}$. Conversely, take any $C \in \bigcup_{s \in S} \sigma_{s}$. Then $C=\sqcup \mathcal{F}$ for some $\mathcal{F} \subseteq \mathcal{B}$. By the definition of atom, $C \in \mathcal{F}$. Hence, $C \in \mathcal{B}$.

Example 2. In Example 1. It is trivial to verify that $\mathcal{C}$ is a fuzzy competence space. We have $\sigma_{s_{1}^{0.6}}=\left\{\left\{\frac{0.6}{s_{1}}, \frac{0.6}{s_{4}}\right\}\right\}, \sigma_{s_{1}^{0.8}}=\left\{\left\{\frac{0.8}{s_{1}}, \frac{0.9}{s_{3}}\right\},\left\{\frac{0.8}{s_{1}}, \frac{1}{s_{2}}, \frac{0.8}{s_{4}}\right\}\right\}, \sigma_{s_{1}^{1}}=\left\{\left\{\frac{1}{s_{1}}, \frac{0.7}{s_{2}}, \frac{1}{s_{3}}\right\},\left\{\frac{1}{s_{1}}, \frac{0.9}{s_{3}}, \frac{1}{s_{4}}\right\}\right\}, \sigma_{s_{2}^{0.7}}=$ $\left\{\left\{\frac{0.7}{s_{2}}\right\}\right\}, \sigma_{s_{2}^{1}}=\left\{\left\{\frac{0.8}{s_{1}}, \frac{1}{s_{2}}, \frac{0.8}{s_{4}}\right\}, \sigma_{s_{3}^{0.9}}=\left\{\left\{\frac{0.8}{s_{1}}, \frac{0.9}{s_{3}}\right\},\left\{\frac{0.7}{s_{2}}, \frac{0.9}{s_{3}}\right\},\left\{\frac{0.9}{s_{3}}, \frac{0.8}{s_{4}}\right\}\right\}, \sigma_{s_{3}^{1}}=\left\{\left\{\frac{1}{s_{1}}, \frac{0.7}{s_{2}}, \frac{1}{s_{3}}\right\}\right\}, \sigma_{s_{4}^{0.6}}=\right.$ $\left\{\left\{\frac{0.6}{s_{1}}, \frac{0.6}{s_{4}}\right\}\right\}, \sigma_{s_{4}^{0.8}}=\left\{\left\{\frac{0.9}{s_{3}}, \frac{0.8}{s_{4}}\right\},\left\{\frac{0.8}{s_{1}}, \frac{1}{s_{2}}, \frac{0.8}{s_{4}}\right\}\right\}, \sigma_{s_{4}^{1}}=\left\{\left\{\frac{1}{s_{1}}, \frac{0.9}{s_{3}}, \frac{1}{s_{4}}\right\}\right\}$. Therefore, the basis of $\mathcal{C}$ is $\mathcal{B}=\left\{\left\{\frac{0.7}{s_{2}}\right\},\left\{\frac{0.6}{s_{1}}, \frac{0.6}{s_{4}}\right\},\left\{\frac{0.8}{s_{1}}, \frac{0.9}{s_{3}}\right\},\left\{\frac{0.7}{s_{2}}, \frac{0.9}{s_{3}}\right\},\left\{\frac{0.9}{s_{3}}, \frac{0.8}{s_{4}}\right\},\left\{\frac{0.8}{s_{1}}, \frac{1}{s_{2}}, \frac{0.8}{s_{4}}\right\},\left\{\frac{1}{s_{1}}, \frac{0.7}{s_{2}}, \frac{1}{s_{3}}\right\},\left\{\frac{1}{s_{1}}, \frac{0.9}{s_{3}}, \frac{1}{s_{4}}\right\}\right\}$.

\section{Competence-based fuzzy skill functions}

Definition 7. (Sun, Li, Ge, \& Lin, 2021) Let $\mu: Q \rightarrow \mathcal{P}^{*}(\mathcal{F}(S) \backslash\{\varnothing\})$ and the elements in $\mu(q)$ are pairwise incomparable for any $q \in Q$. The triple $(Q, S, \mu)$ is called a fuzzy skill function.

It's obvious that each element in $\mu(q)$ represents a minimal competency for solving the item $q$.

Definition 8. Let $\left(S, V_{s}, \mathcal{C}\right)$ be a fuzzy competence structure, $(Q, S, \mu)$ be a fuzzy skill function and $T \in \mathcal{C}$. If $K=\{q \in Q \mid C \subseteq T$ for some $C \in \mu(q)\}$, then $K$ is said to be the knowledge state delineated by $T \in \mathcal{C}$ via fuzzy skill function $(Q, S, \mu)$.

Definition 9. Let $\left(S, V_{s}, \mathcal{C}\right)$ be a fuzzy competence structure and $(Q, S, \mu)$ be a fuzzy skill function. Define a mapping $p_{\mu}: \mathcal{C} \rightarrow \mathcal{P}(Q)$ by

$$
p_{\mu}(T)=\{q \in Q \mid C \subseteq T \text { for some } C \in \mu(q)\} .
$$

The triple $\left(Q, S, p_{\mu}\right)$ is said to be the problem function of $(Q, S, \mu)$.

Sometimes, a problem function $\left(Q, S, p_{\mu}\right)$ is denoted by $p_{\mu}$ for convenience. Obviously, $p_{\mu}(S \times$ $\{0\})=\varnothing$ and $p_{\mu}(S \times\{1\})=Q$. Thus, the set $\left\{p_{\mu}(T) \mid T \in \mathcal{C}\right\}$ is a knowledge structure, which is said to be the knowledge structure delineated via competence-based fuzzy skill function $(Q, S, \mu)$.

Lemma 1. For any fuzzy competence structure $\left(S, V_{s}, \mathcal{C}\right)$ and any fuzzy skill function $(Q, S, \mu)$, the problem function $p_{\mu}$ is monotonic.

Proof. Let $T_{1}, T_{2} \in \mathcal{C}, T_{1} \subseteq T_{2}$ and $q \in p_{\mu}\left(T_{1}\right)$. Then there exists $C \in \mu(q)$ such that $C \subseteq T_{1} \subseteq T_{2}$. Thus, $q \in p_{\mu}\left(T_{2}\right), p_{\mu}\left(T_{1}\right) \subseteq p_{\mu}\left(T_{2}\right)$. 


\subsection{Competence-based disjunctive fuzzy skill functions}

Now, we study knowledge structures induced by competence-based disjunctive fuzzy skill functions.

Definition 10. (Sun, $\mathrm{Li}, \mathrm{Ge}, \& \mathrm{Lin}, 2021)$ A fuzzy skill function $(Q, S, \mu)$ is said to be disjunctive if for any $C \in \mu(q)$ and $q \in Q$ there exists unique $s \in S$ such that $C(s) \neq 0$.

Remark 2. Sun et al. (2021) showed that via the disjunctive model, fuzzy skill maps delineate knowledge spaces. Before that, Heller et al. (2013) proved that for competence spaces, disjunctive skill functions delineate knowledge spaces.

Proposition 1. Let $\left(S, V_{s}, \mathcal{C}\right)$ be a fuzzy competence space, $(Q, S, \mu)$ be a disjunctive fuzzy skill function and $\mathcal{K}$ the corresponding knowledge structure. Then $\mathcal{K}$ is a knowledge space.

Proof. Take any $K_{1}, K_{2} \in \mathcal{K}$. Assume that $K_{i}$ is delineated by $T_{i} \in \mathcal{C}$ for any $i=1,2$. Consider any $q \in K_{1} \cup K_{2}$. There exists $s \in S$ and $C_{q} \in \mu(q)$ such that $0<C_{q}(s) \leq T_{1}(s)$ or $0<C_{q}(s) \leq T_{2}(s)$. Thus, $0<C_{q}(s) \leq\left(T_{1} \sqcup T_{2}\right)(s)$. It follows that $K_{1} \cup K_{2}$ is delineated by $T_{1} \sqcup T_{2}$. Then $K_{1} \cup K_{2} \in \mathcal{K}$, since $T_{1} \sqcup T_{2} \in \mathcal{C}$. Therefore, $(Q, \mathcal{K})$ is a knowledge space.

Remark 3. It is trivial to verify that the corresponding conclusion of Proposition 1 may not hold if the fuzzy skill function $(Q, S, \mu)$ is not disjunctive. Moreover, the corresponding result of Proposition 1 is unnecessarily correct if $\left(S, V_{s}, \mathcal{C}\right)$ is not a fuzzy competence space. Let $Q=\left\{q_{1}, q_{2}, q_{3}\right\}$, $S=\left\{s_{1}, s_{2}, s_{3}\right\}$ and $V_{s_{1}}=\{0,0.8,1\}, V_{s_{2}}=\{0,0.7,1\}, V_{s_{3}}=\{0,0.7,1\}$. Consider fuzzy competence structure $\mathcal{C}=\left\{\varnothing,\left\{\frac{0.8}{s_{1}}\right\},\left\{\frac{0.7}{s_{2}}\right\},\left\{\frac{0.8}{s_{1}}, \frac{0.7}{s_{2}}, \frac{0.7}{s_{3}}\right\},\left\{\frac{1}{s_{1}}, \frac{1}{s_{2}}, \frac{1}{s_{3}}\right\}\right\}$ and the disjunctive fuzzy skill function $\mu\left(q_{1}\right)=\left\{\left\{\frac{0.7}{s_{1}}\right\},\left\{\frac{0.7}{s_{3}}\right\}\right\}, \mu\left(q_{2}\right)=\left\{\left\{\frac{0.6}{s_{2}}\right\},\left\{\frac{0.8}{s_{3}}\right\}\right\}, \mu\left(q_{3}\right)=\left\{\left\{\frac{0.6}{s_{3}}\right\}\right\}$. It's easy to see that $\mathcal{C}$ is not a fuzzy competence space. Note that $p_{\mu}(\varnothing)=\varnothing, p_{\mu}\left(\left\{\frac{0.8}{s_{1}}\right\}\right)=\left\{q_{1}\right\}, p_{\mu}\left(\left\{\frac{0.7}{s_{2}}\right\}\right)=\left\{q_{2}\right\}$, $p_{\mu}\left(\left\{\frac{0.8}{s_{1}}, \frac{0.7}{s_{2}}, \frac{0.7}{s_{3}}\right\}\right)=\{Q\}, p_{\mu}\left(\left\{\frac{1}{s_{1}}, \frac{1}{s_{2}}, \frac{1}{s_{3}}\right\}\right)=\{Q\}$. Thus, the knowledge structure induced by the competence-based disjunctive fuzzy skill function is $\mathcal{K}=\left\{\varnothing,\left\{q_{1}\right\},\left\{q_{2}\right\}, Q\right\}$. Obviously, $\mathcal{K}$ is not a knowledge space.

Lemma 2. Let $\left(S, V_{s}, \mathcal{C}\right)$ be a fuzzy competence space and $p_{\mu}: \mathcal{C} \rightarrow \mathcal{P}(Q)$ is the problem function of a disjunctive fuzzy skill function $(Q, S, \mu)$. Then $p_{\mu}\left(T_{1} \sqcup T_{2}\right)=p_{\mu}\left(T_{1}\right) \cup p_{\mu}\left(T_{2}\right)$ for all $T_{1}, T_{2} \in \mathcal{C}$.

Proof. For any $T_{1}, T_{2} \in \mathcal{C}$, by Lemma 1 , we have $p_{\mu}\left(T_{1}\right) \cup p_{\mu}\left(T_{2}\right) \subseteq p_{\mu}\left(T_{1} \sqcup T_{2}\right)$. Let $q \in p_{\mu}\left(T_{1} \sqcup T_{2}\right)$. Then there exists $C \in \mu(q)$ such that $C \subseteq T_{1} \sqcup T_{2}$. It follows that $C \subseteq T_{1}$ or $C \subseteq T_{2}$ since $C(s) \neq 0$ for unique $s \in S$. Hence, $q \in p_{\mu}(C) \subseteq p_{\mu}\left(T_{1}\right) \cup p_{\mu}\left(T_{2}\right)$.

Remark 4. The reverse of Lemma 2 is unnecessarily correct. Let $Q=\left\{q_{1}, q_{2}, q_{3}\right\}, S=\left\{s_{1}, s_{2}, s_{3}\right\}$ and $V_{s_{1}}=\{0,0.8,1\}, V_{s_{2}}=\{0,0.7,1\}, V_{s_{3}}=\{0,0.7,1\}$. Consider the fuzzy competence space $\mathcal{C}=\left\{\varnothing,\left\{\frac{0.8}{s_{1}}\right\},\left\{\frac{0.7}{s_{2}}\right\},\left\{\frac{0.8}{s_{1}}, \frac{0.7}{s_{2}}\right\},\left\{\frac{0.8}{s_{1}}, \frac{0.7}{s_{2}}, \frac{0.7}{s_{3}}\right\},\left\{\frac{1}{s_{1}}, \frac{1}{s_{2}}, \frac{1}{s_{3}}\right\}\right\}$ and the fuzzy skill function $(Q, S, \mu)$ is defined as: $\mu\left(q_{1}\right)=\left\{\left\{\frac{0.8}{s_{1}}\right\}\right\}, \mu\left(q_{2}\right)=\left\{\left\{\frac{0.7}{s_{2}}\right\}\right\}, \mu\left(q_{3}\right)=\left\{\left\{\frac{0.8}{s_{1}}, \frac{0.7}{s_{2}}, \frac{0.7}{s_{3}}\right\}\right\}$. It is easy to verify that $p_{\mu}\left(T_{1} \sqcup T_{2}\right)=p_{\mu}\left(T_{1}\right) \cup p_{\mu}\left(T_{2}\right)$ for all $T_{1}, T_{2} \in \mathcal{C}$. However, $(Q, S, \mu)$ is not disjunctive.

Theorem 2. For any fuzzy competence space $\left(S, V_{s}, \mathcal{C}\right)$ and disjunctive fuzzy skill function $(Q, S, \mu)$, the knowledge space $\mathcal{K}$ delineated by $\mu$ is the span of $\left\{p_{\mu}(B) \mid B \in \mathcal{B}\right\}$, where $\mathcal{B}$ is the basis of $\left(S, V_{s}, \mathcal{C}\right)$. 
Proof. Take any $K \in \mathcal{K}$, and consider a competence state $T \in \mathcal{C}$ that delineates it. Denote $T=\sqcup \mathcal{B}^{\prime}$ for some $\mathcal{B}^{\prime} \subseteq \mathcal{B}$, since $\mathcal{B}$ is the basis of $\left(S, V_{s}, \mathcal{C}\right)$. For any $q \in K$, there exists $C_{q} \in \mu(q)$ such that $C_{q} \subseteq T$. For any $B \in \mathcal{B}^{\prime}$, by Definition $9, p_{\mu}(B) \in \mathcal{K}$. According to Lemma 2, $p_{\mu}(T)=p_{\mu}\left(\sqcup_{B \in \mathcal{B}^{\prime}} B\right)=\bigcup_{B \in \mathcal{B}^{\prime}} p_{\mu}(B)$. It follows that $K=\bigcup_{B \in \mathcal{B}^{\prime}} p_{\mu}(B)$. Consequently, $(Q, \mathcal{K})$ is the span of $\left\{p_{\mu}(B) \mid B \in \mathcal{B}\right\}$.

Example 3. In Example 1, let $Q=\left\{q_{1}, q_{2}, q_{3}, q_{4}\right\}$. The fuzzy competence space $\mathcal{C}$ on $S$ is given in Table 2. The basis of $\left(S, V_{s}, \mathcal{C}\right)$ is $\mathcal{B}=\left\{\left\{\frac{0.7}{s_{2}}\right\},\left\{\frac{0.6}{s_{1}}, \frac{0.6}{s_{4}}\right\},\left\{\frac{0.8}{s_{1}}, \frac{0.9}{s_{3}}\right\},\left\{\frac{0.7}{s_{2}}, \frac{0.9}{s_{3}}\right\},\left\{\frac{0.9}{s_{3}}, \frac{0.8}{s_{4}}\right\},\left\{\frac{0.8}{s_{1}}, \frac{1}{s_{2}}, \frac{0.8}{s_{4}}\right\}\right.$, $\left.\left\{\frac{1}{s_{1}}, \frac{0.7}{s_{2}}, \frac{1}{s_{3}}\right\},\left\{\frac{1}{s_{1}}, \frac{0.9}{s_{3}}, \frac{1}{s_{4}}\right\}\right\}$. A disjunctive fuzzy skill function $(Q, S, \mu)$ is defined as: $\mu\left(q_{1}\right)=$ $\left\{\left\{\frac{0.6}{s_{1}}\right\},\left\{\frac{0.8}{s_{2}}\right\}\right\}, \mu\left(q_{2}\right)=\left\{\left\{\frac{0.7}{s_{2}}\right\}\right\}, \mu\left(q_{3}\right)=\left\{\left\{\frac{0.7}{s_{4}}\right\}\right\}, \mu\left(q_{4}\right)=\left\{\left\{\frac{0.7}{s_{1}}\right\},\left\{\frac{0.9}{s_{3}}\right\},\left\{\frac{0.8}{s_{4}}\right\}\right\}$, see Table 3 . According to Theorem 2, the span of $\left\{p_{\mu}(B) \mid B \in \mathcal{B}\right\}$ is the knowledge space induced by the

Table 3: Item-skill table of the disjunctive fuzzy skill function $(Q, S, \mu)$

\begin{tabular}{ccccc}
\hline & $s_{1}$ & $s_{2}$ & $s_{3}$ & $s_{4}$ \\
\hline$q_{1}$ & 0.6 & 0 & 0 & 0 \\
& 0 & 0.8 & 0 & 0 \\
$q_{2}$ & 0 & 0.7 & 0 & 0 \\
$q_{3}$ & 0 & 0 & 0 & 0.7 \\
$q_{4}$ & 0.7 & 0 & 0 & 0 \\
& 0 & 0 & 0.9 & 0 \\
& 0 & 0 & 0 & 0.8 \\
\hline
\end{tabular}

competence-based disjunctive fuzzy skill function. Note that $p_{\mu}\left(\left\{\frac{0.7}{s_{2}}\right\}\right)=\left\{q_{2}\right\}, p_{\mu}\left(\left\{\frac{0.6}{s_{1}}, \frac{0.6}{s_{4}}\right\}\right)=$ $\left\{q_{1}\right\}, p_{\mu}\left(\left\{\frac{0.8}{s_{1}}, \frac{0.9}{s_{3}}\right\}\right)=\left\{q_{1}, q_{4}\right\}, p_{\mu}\left(\left\{\frac{0.7}{s_{2}}, \frac{0.9}{s_{3}}\right\}\right)=\left\{q_{2}, q_{4}\right\}, p_{\mu}\left(\left\{\frac{0.9}{s_{3}}, \frac{0.8}{s_{4}}\right\}\right)=\left\{q_{3}, q_{4}\right\}, p_{\mu}\left(\left\{\frac{0.8}{s_{1}}, \frac{1}{s_{2}}, \frac{0.8}{s_{4}}\right\}\right)=$ $\{Q\}, p_{\mu}\left(\left\{\frac{1}{s_{1}}, \frac{0.7}{s_{2}}, \frac{1}{s_{3}}\right\}\right)=\left\{q_{1}, q_{2}, q_{4}\right\}, p_{\mu}\left(\left\{\frac{1}{s_{1}}, \frac{0.9}{s_{3}}, \frac{1}{s_{4}}\right\}\right)=\left\{q_{1}, q_{3}, q_{4}\right\}$. Then $\mathcal{K}$ is the span of

$$
\left\{\left\{q_{1}\right\},\left\{q_{2}\right\},\left\{q_{1}, q_{4}\right\},\left\{q_{2}, q_{4}\right\},\left\{q_{3}, q_{4}\right\},\left\{q_{1}, q_{2}, q_{4}\right\},\left\{q_{1}, q_{3}, q_{4}\right\}, Q\right\} .
$$

Eventually,

$$
\mathcal{K}=\left\{\varnothing,\left\{q_{1}\right\},\left\{q_{2}\right\},\left\{q_{1}, q_{2}\right\},\left\{q_{1}, q_{4}\right\},\left\{q_{2}, q_{4}\right\},\left\{q_{3}, q_{4}\right\},\left\{q_{1}, q_{2}, q_{4}\right\},\left\{q_{1}, q_{3}, q_{4}\right\},\left\{q_{2}, q_{3}, q_{4}\right\}, Q\right\} .
$$

By the Theorem 2, we design an algorithm that delineates knowledge spaces via competencebased disjunctive fuzzy skill functions, which is only concerned with the bases of fuzzy competence spaces without listing all fuzzy competence states of fuzzy competence spaces.

Let $(Q, S, \mu)$ be a disjunctive fuzzy skill function, $\left(S, V_{s}, \mathcal{C}\right)$ a fuzzy competence space and $\mathcal{B}$ the basis of $\left(S, V_{s}, \mathcal{C}\right)$. The algorithm is as follows.

Step 1. Compute $p_{\mu}(B)$ for each atom $B \in \mathcal{B}$.

Step 2. Compute $\mathbb{S}\left(\left\{p_{\mu}(B) \mid B \in \mathcal{B}\right\}\right)$, then $\mathcal{K}=\mathbb{S}\left(\left\{p_{\mu}(B) \mid B \in \mathcal{B}\right\}\right)$.

\subsection{Competence-based conjunctive fuzzy skill functions}

Now, we study knowledge structures induced by competence-based conjunctive fuzzy skill functions.

Definition 11. (Sun, Li, Ge, \& Lin, 2021) A fuzzy skill function $(Q, S, \mu)$ is said to be conjunctive if for any $q \in Q, \mu(q)=\{C\}$ for some $C \in \mathcal{F}(S) \backslash\{\varnothing\}$. 
Proposition 2. Let $\left(S, V_{s}, \mathcal{C}\right)$ be a $\sqcap$-closed fuzzy competence structure, $(Q, S, \mu)$ be a conjunctive fuzzy skill function and $\mathcal{K}$ the corresponding knowledge structure. Then $\mathcal{K}$ is a simple closure space.

Proof. Take any $K_{1}, K_{2} \in \mathcal{K}$. Assume that $K_{i}$ is delineated by $T_{i} \in \mathcal{C}$ for any $i=1,2$. For any $q \in K_{1} \cap K_{2}$, there exists unique $C_{q} \in \mu(q)$ such that $C_{q} \subseteq T_{1}$ and $C_{q} \subseteq T_{2}$. Then $C_{q} \subseteq T_{1} \sqcap T_{2}$. It follows that $K_{1} \cap K_{2}$ is delineated by $T_{1} \sqcap T_{2}$. Then $K_{1} \cap K_{2} \in \mathcal{K}$, since $T_{1} \sqcap T_{2} \in \mathcal{C}$. Therefore, $(Q, \mathcal{K})$ is a simple closure space.

Remark 5. Proposition 2 is unnecessarily correct if $\left(S, V_{s}, \mathcal{C}\right)$ is not $\sqcap$-closed. Consider the following example. Let $Q=\left\{q_{1}, q_{2}, q_{3}\right\}, S=\left\{s_{1}, s_{2}, s_{3}\right\}, V_{s_{1}}=\{0,0.8,1\}, V_{s_{2}}=\{0,0.7,1\}, V_{s_{3}}=\{0,0.7,1\}$ and fuzzy competence structure $\mathcal{C}=\left\{\varnothing,\left\{\frac{0.8}{s_{1}}, \frac{0.7}{s_{2}}\right\},\left\{\frac{0.7}{s_{2}}, \frac{0.7}{s_{3}}\right\},\left\{\frac{1}{s_{1}}, \frac{1}{s_{2}}, \frac{1}{s_{3}}\right\}\right\}$. A conjunctive fuzzy skill function is defined as $\mu\left(q_{1}\right)=\left\{\left\{\frac{0.7}{s_{1}}, \frac{0.6}{s_{2}}\right\}\right\}, \mu\left(q_{2}\right)=\left\{\left\{\frac{0.7}{s_{2}}\right\}\right\}, \mu\left(q_{3}\right)=\left\{\left\{\frac{0.6}{s_{2}}, \frac{0.6}{s_{3}}\right\}\right\}$. It is clear that $\mathcal{C}$ is not a $\sqcap$-closed fuzzy competence structure. We have $p_{\mu}(\varnothing)=\varnothing, p_{\mu}\left(\left\{\frac{0.8}{s_{1}}, \frac{0.7}{s_{2}}\right\}\right)=$ $\left\{q_{1}, q_{2}\right\}, p_{\mu}\left(\left\{\frac{0.7}{s_{2}}, \frac{0.7}{s_{3}}\right\}\right)=\left\{q_{2}, q_{3}\right\}, p_{\mu}\left(\left\{\frac{1}{s_{1}}, \frac{1}{s_{2}}, \frac{1}{s_{3}}\right\}\right)=\{Q\}$. The knowledge structure induced by the competence-based conjunctive fuzzy skill function is $\mathcal{K}=\left\{\varnothing,\left\{q_{1}, q_{2}\right\},\left\{q_{2}, q_{3}\right\}, Q\right\}$. Obviously, $\mathcal{K}$ is not a simple closure space.

Lemma 3. Let $\left(S, V_{s}, \mathcal{C}\right)$ be a $\sqcap$-closed fuzzy competence structure and $p_{\mu}: \mathcal{C} \rightarrow \mathcal{P}(Q)$ is the problem function of a conjunctive fuzzy skill function $(Q, S, \mu)$. Then $p_{\mu}\left(T_{1} \sqcap T_{2}\right)=p_{\mu}\left(T_{1}\right) \cap p_{\mu}\left(T_{2}\right)$ for any $T_{1}, T_{2} \in \mathcal{C}$.

Proof. For any $T_{1}, T_{2} \in \mathcal{C}$, according to Lemma 1, we have $p_{\mu}\left(T_{1} \sqcap T_{2}\right) \subseteq p_{\mu}\left(T_{1}\right) \cap p_{\mu}\left(T_{2}\right)$. Let $q \in p_{\mu}\left(T_{1}\right) \cap p_{\mu}\left(T_{2}\right)$. We have $C \subseteq T_{1}$ and $C \subseteq T_{2}$, since $\mu(q)=\{C\}$. Thus, $C \subseteq T_{1} \sqcap T_{2}$. It follows that $q \in p_{\mu}(C) \subseteq p_{\mu}\left(T_{1} \sqcap T_{2}\right)$.

Remark 6 . The reverse of Lemma 3 is unnecessarily correct. Consider the following example. Let $Q=\left\{q_{1}, q_{2}, q_{3}\right\}, S=\left\{s_{1}, s_{2}, s_{3}\right\}$ and $V_{s_{1}}=\{0,0.8,1\}, V_{s_{2}}=\{0,0.7,1\}, V_{s_{3}}=\{0,0.7,1\}$. Consider the $\sqcap$-closed fuzzy competence structure $\mathcal{C}=\left\{\varnothing,\left\{\frac{0.7}{s_{2}}\right\},\left\{\frac{0.8}{s_{1}}, \frac{0.7}{s_{2}}\right\},\left\{\frac{0.7}{s_{2}}, \frac{0.7}{s_{3}}\right\},\left\{\frac{1}{s_{1}}, \frac{1}{s_{2}}, \frac{1}{s_{3}}\right\}\right\}$ and the fuzzy skill function $(Q, S, \mu)$ is defined as: $\mu\left(q_{1}\right)=\left\{\left\{\frac{0.8}{s_{1}}, \frac{0.7}{s_{2}}\right\}\right\}, \mu\left(q_{2}\right)=\left\{\left\{\frac{0.7}{s_{2}}\right\},\left\{\frac{0.8}{s_{1}}, \frac{0.6}{s_{2}}\right\},\left\{\frac{0.6}{s_{2}}, \frac{0.7}{s_{3}}\right\}\right\}$, $\mu\left(q_{3}\right)=\left\{\left\{\frac{0.7}{s_{2}}, \frac{0.7}{s_{3}}\right\}\right\}$. It is easy to verify that $p_{\mu}\left(T_{1} \sqcap T_{2}\right)=p_{\mu}\left(T_{1}\right) \cap p_{\mu}\left(T_{2}\right)$ for all $T_{1}, T_{2} \in \mathcal{C}$. However, $(Q, S, \mu)$ is not conjunctive.

Definition 12. (Sun, Li, Ge, \& Lin, 2021) Let $(Q, \mathcal{K})$ be a simple closure space. The collection $\mathcal{K}^{\prime} \subseteq \mathcal{K}$ is said to be an intersection generation group of $\mathcal{K}$ if for any $K \in \mathcal{K}$, there exists $\mathcal{A} \subseteq \mathcal{K}^{\prime}$ such that $K=\bigcap \mathcal{A}$, written as $\mathbb{I}\left(\mathcal{K}^{\prime}\right)=\mathcal{K}$.

Similarity, the concept of intersection generation group can be extend to fuzzy competence structure. Let $\left(S, V_{s}, \mathcal{C}\right)$ be a $\sqcap$-closed fuzzy competence structure. The collection $\mathcal{C}^{\prime} \subseteq \mathcal{C}$ is said to be an intersection generation group of $\mathcal{C}$ if for every $C \in \mathcal{C}$, there exists $\mathcal{A} \subseteq \mathcal{C}^{\prime}$ such that $C=\sqcap \mathcal{A}$, written as $\mathbb{I}\left(\mathcal{C}^{\prime}\right)=\mathcal{C}$.

Theorem 3. Let $\left(S, V_{s}, \mathcal{C}\right)$ be a $\sqcap$-closed fuzzy competence structure and $\mathcal{K}$ be the knowledge structure delineated by a conjunctive fuzzy skill function $(Q, S, \mu)$. Then $\mathcal{K}=\mathbb{I}\left(\left\{p_{\mu}(A) \mid A \in \mathcal{A}\right\}\right)$, where $\mathcal{A}$ is the minimal intersection generation group of $\left(S, V_{s}, \mathcal{C}\right)$.

Proof. Take any $K \in \mathcal{K}$, and consider a competence state $T \in \mathcal{C}$ that exactly delineates it. For any item $q \in K$ and $\mu(q)=\{C\}$, we have $C \subseteq T$. Moreover $T=\sqcap \mathcal{A}^{\prime}$ for some $\mathcal{A}^{\prime} \subseteq \mathcal{A}$, since $\mathbb{I}(\mathcal{A})=\mathcal{C}$. For any $A \in \mathcal{A}^{\prime}, p_{\mu}(A) \in \mathcal{K}$. According to Lemma $3, p_{\mu}(T)=p_{\mu}\left(\sqcap_{A \in \mathcal{A}^{\prime}} A\right)=\bigcap_{A \in \mathcal{A}^{\prime}} p_{\mu}(A)$. It follows that $K=\bigcap_{A \in \mathcal{A}^{\prime}} p_{\mu}(A)$. Consequently, $\mathcal{K}=\mathbb{I}\left(\left\{p_{\mu}(A) \mid A \in \mathcal{A}\right\}\right)$. 
Example 4. In Example 1. Consider the fuzzy competence structure $\left(S, V_{s}, \mathcal{C}\right)$ in Table 4 . It is trivial to verify that $\mathcal{C}$ is a $\sqcap$-closed fuzzy competence structure. The minimal intersection gener-

Table 4: A $\sqcap$-closed fuzzy competence structure $\left(S, V_{s}, \mathcal{C}\right)$

\begin{tabular}{cccccccccc}
\hline & $s_{1}$ & $s_{2}$ & $s_{3}$ & $s_{4}$ & & $s_{1}$ & $s_{2}$ & $s_{3}$ & $s_{4}$ \\
\hline$C_{1}$ & 0 & 0 & 0 & 0 & $C_{16}$ & 0.6 & 0.7 & 0.9 & 0.6 \\
$C_{2}$ & 0 & 0 & 0.9 & 0 & $C_{17}$ & 0.6 & 0.7 & 0.9 & 0.8 \\
$C_{3}$ & 0 & 0 & 1 & 0 & $C_{18}$ & 0.6 & 0.7 & 1 & 0.6 \\
$C_{4}$ & 0.6 & 0 & 0.9 & 0 & $C_{19}$ & 0.6 & 0.7 & 1 & 0.8 \\
$C_{5}$ & 0.6 & 0 & 1 & 0 & $C_{20}$ & 0.6 & 0.7 & 0.9 & 1 \\
$C_{6}$ & 0.8 & 0 & 0.9 & 0 & $C_{21}$ & 0.6 & 0.7 & 1 & 1 \\
$C_{7}$ & 0.8 & 0 & 1 & 0 & $C_{22}$ & 0.8 & 0.7 & 0.9 & 0.6 \\
$C_{8}$ & 0 & 0 & 0.9 & 0.6 & $C_{23}$ & 0.8 & 0.7 & 1 & 0.6 \\
$C_{9}$ & 0 & 0.7 & 0.9 & 0.6 & $C_{24}$ & 0.8 & 0.7 & 0.9 & 0.8 \\
$C_{10}$ & 0 & 0.7 & 1 & 0.6 & $C_{25}$ & 0.8 & 0.7 & 1 & 0.8 \\
$C_{11}$ & 0 & 0.7 & 0.9 & 0.8 & $C_{26}$ & 0.8 & 0.7 & 0.9 & 1 \\
$C_{12}$ & 0 & 0.7 & 1 & 0.8 & $C_{27}$ & 0.8 & 1 & 0.9 & 1 \\
$C_{13}$ & 0.6 & 0 & 0.9 & 0.6 & $C_{28}$ & 1 & 0.7 & 0.9 & 1 \\
$C_{14}$ & 0.8 & 0 & 0.9 & 0.6 & $C_{29}$ & 1 & 1 & 1 & 1 \\
$C_{15}$ & 1 & 0 & 0.9 & 0.6 & & & & & \\
\hline
\end{tabular}

ation group of $\left(S, V_{s}, \mathcal{C}\right)$ is

$$
\begin{aligned}
\mathcal{A}= & \left\{\left\{\frac{0.8}{s_{1}}, \frac{1}{s_{3}}\right\},\left\{\frac{0.7}{s_{2}}, \frac{1}{s_{3}}, \frac{0.8}{s_{4}}\right\},\left\{\frac{1}{s_{1}}, \frac{0.9}{s_{3}}, \frac{0.6}{s_{4}}\right\},\left\{\frac{0.6}{s_{1}}, \frac{0.7}{s_{2}}, \frac{1}{s_{3}}, \frac{1}{s_{4}}\right\},\left\{\frac{0.8}{s_{1}}, \frac{0.7}{s_{2}}, \frac{1}{s_{3}}, \frac{0.6}{s_{4}}\right\},\right. \\
& \left.\left\{\frac{0.8}{s_{1}}, \frac{0.7}{s_{2}}, \frac{1}{s_{3}}, \frac{0.8}{s_{4}}\right\},\left\{\frac{0.8}{s_{1}}, \frac{1}{s_{2}}, \frac{0.9}{s_{3}}, \frac{1}{s_{4}}\right\},\left\{\frac{1}{s_{1}}, \frac{0.7}{s_{2}}, \frac{0.9}{s_{3}}, \frac{1}{s_{4}}\right\},\left\{\frac{1}{s_{1}}, \frac{1}{s_{2}}, \frac{1}{s_{3}}, \frac{1}{s_{4}}\right\}\right\} .
\end{aligned}
$$

A conjunctive fuzzy skill function $(Q, S, \mu)$ is defined as: $\mu\left(q_{1}\right)=\left\{\left\{\frac{1}{s_{1}}, \frac{0.9}{s_{3}}\right\}\right\}, \mu\left(q_{2}\right)=\left\{\left\{\frac{0.8}{s_{1}}, \frac{0.8}{s_{2}}, \frac{0.8}{s_{3}}\right\}\right\}$, $\mu\left(q_{3}\right)=\left\{\left\{\frac{0.8}{s_{4}}\right\}\right\}, \mu\left(q_{4}\right)=\left\{\left\{\frac{1}{s_{1}}, \frac{0.9}{s_{3}}, \frac{0.9}{s_{4}}\right\}\right\}$. According to Theorem 3, the knowledge structure induced by $\mu$ is $\mathbb{I}\left(\left\{p_{\mu}(A) \mid A \in \mathcal{A}\right\}\right)$. Moreover,

$$
\begin{aligned}
& p_{\mu}\left(\left\{\frac{0.8}{s_{1}}, \frac{1}{s_{3}}\right\}\right)=\varnothing, \\
& p_{\mu}\left(\left\{\frac{0.7}{s_{2}}, \frac{1}{s_{3}}, \frac{0.8}{s_{4}}\right\}\right)=\left\{q_{3}\right\}, \\
& p_{\mu}\left(\left\{\frac{1}{s_{1}}, \frac{0.9}{s_{3}}, \frac{0.6}{s_{4}}\right\}\right)=\left\{q_{1}\right\}, \\
& p_{\mu}\left(\left\{\frac{0.6}{s_{1}}, \frac{0.7}{s_{2}}, \frac{1}{s_{3}}, \frac{1}{s_{4}}\right\}\right)=\left\{q_{3}\right\}, \\
& p_{\mu}\left(\left\{\frac{0.8}{s_{1}}, \frac{0.7}{s_{2}}, \frac{1}{s_{3}}, \frac{0.6}{s_{4}}\right\}\right)=\{\varnothing\}, \\
& p_{\mu}\left(\left\{\frac{0.8}{s_{1}}, \frac{0.7}{s_{2}}, \frac{1}{s_{3}}, \frac{0.8}{s_{4}}\right\}\right)=\left\{q_{3}\right\}, \\
& p_{\mu}\left(\left\{\frac{0.8}{s_{1}}, \frac{1}{s_{2}}, \frac{0.9}{s_{3}}, \frac{1}{s_{4}}\right\}\right)=\left\{q_{2}, q_{3}\right\}, \\
& p_{\mu}\left(\left\{\frac{1}{s_{1}}, \frac{0.7}{s_{2}}, \frac{0.9}{s_{3}}, \frac{1}{s_{4}}\right\}\right)=\left\{q_{1}, q_{3}, q_{4}\right\}, \\
& \left.p_{\mu}\left(\left\{\frac{1}{s_{1}}, \frac{1}{s_{2}}, \frac{1}{s_{3}}, \frac{1}{s_{4}}\right\}\right\}\right)=Q .
\end{aligned}
$$

Eventually,

$$
\mathcal{K}=\mathbb{I}\left(\left\{\varnothing,\left\{q_{1}\right\},\left\{q_{3}\right\},\left\{q_{2}, q_{3}\right\},\left\{q_{1}, q_{3}, q_{4}\right\}, Q\right\}\right)=\left\{\varnothing,\left\{q_{1}\right\},\left\{q_{3}\right\},\left\{q_{2}, q_{3}\right\},\left\{q_{1}, q_{3}, q_{4}\right\}, Q\right\} .
$$

By the Theorem 3, we design an algorithm that delineates simple closure spaces via competencebased conjunctive fuzzy skill functions, which is only concerned with the minimal intersection generation groups of $\sqcap$-closed fuzzy competence structures.

Let $(Q, S, \mu)$ be a conjunctive fuzzy skill function, $\left(S, V_{s}, \mathcal{C}\right)$ a $\sqcap$-closed fuzzy competence structure and $\mathcal{A}$ the minimal intersection generation group of $\left(S, V_{s}, \mathcal{C}\right)$. The algorithm is as follows. 
Step 1. Compute $p_{\mu}(A)$ for each $A \in \mathcal{A}$.

Step 2. Compute $\mathbb{I}\left(\left\{p_{\mu}(A) \mid A \in \mathcal{A}\right\}\right)$, then $\mathcal{K}=\mathbb{I}\left(\left\{p_{\mu}(A) \mid A \in \mathcal{A}\right\}\right)$.

\subsection{Competence-based fuzzy skill functions}

Now, we study knowledge structures delineated via competence-based fuzzy skill functions.

Definition 13. (Sun, $\mathrm{Li}, \mathrm{Ge}, \& \mathrm{Lin}, 2021)$ For a fuzzy skill function $(Q, S, \mu)$, an element $C^{\prime} \in \mathcal{F}(S)$ is said to be a weak competency if $C^{\prime} \notin \bigcup_{q \in Q} \mu(q)$ and $C^{\prime}=\bigsqcup \mathcal{A}$ for some $\mathcal{A} \subseteq \bigcup_{q \in Q} \mu(q)$.

For a fuzzy skill function $(Q, S, \mu)$, the collection of all competencies and all weak competencies of $(Q, S, \mu)$ is denoted by $\mathcal{H}$.

Definition 14. Given a fuzzy competence structure $\left(S, V_{s}, \mathcal{C}\right)$ and a fuzzy skill function $(Q, S, \mu)$. For each $H \in \mathcal{H}$, an element $C \in \mathcal{C}$ is said to be an effective fuzzy competence state of $H$ if it satisfies:

(i) $H \subseteq C$;

(ii) for any $C^{\prime} \in \mathcal{C}, H \subseteq C^{\prime}$ and $C^{\prime} \subseteq C$ imply $C=C^{\prime}$.

Remark 7. For any fuzzy skill function $(Q, S, \mu)$ and any fuzzy competence structure $\left(S, V_{s}, \mathcal{C}\right), \varnothing$ is an effective fuzzy competence state, since $\varnothing$ is a weak competency and $\varnothing \in \mathcal{C}$. Note that the collection $\mathcal{H}$ only depends on $(Q, S, \mu)$. Therefore, all effective fuzzy competence states of $\mathcal{H}$ are also called effective fuzzy competence states of $(Q, S, \mu)$. In particular, if the fuzzy competence structure $\mathcal{C}=\mathcal{F}(S)$, then the family of effective fuzzy competence states is the family of competencies and weak competencies.

Theorem 4. For a fuzzy competence structure $\left(S, V_{s}, \mathcal{C}\right)$ and a fuzzy skill function $(Q, S, \mu)$, let $(Q, \mathcal{K})$ be the knowledge structure delineated by $(Q, S, \mu)$. Then each $K \in \mathcal{K}$ can be delineated by an effective fuzzy competence state of $\mu$.

Proof. Denote $\mathcal{H}_{K}=\left\{\bigcup_{q \in K} C_{q} \mid C_{q} \in \mu(q)\right\}$. Take $H \in \mathcal{H}_{K}$ such that if $H^{\prime} \in \mathcal{H}_{K}$ and $H^{\prime} \subseteq H$ implies $H^{\prime}=H$. Let $T$ be an effective fuzzy competence state of $H$. Then, for any $q \in K$, there exists $C_{q} \in \mu(q)$ such that $C_{q} \subseteq T$. Thus, $T$ delineates $K$.

It's obvious that the family of effective fuzzy competence states of $(Q, S, \mu)$ is a subfamily of $\mathcal{C}$, and the number of effective fuzzy competence states is much smaller than $|\mathcal{C}|$. For any fuzzy competence structure $\left(S, V_{s}, \mathcal{C}\right)$ and fuzzy skill function $(Q, S, \mu)$, by Theorem 4 , we build an algorithm that delineates knowledge structures via competence-based fuzzy skill functions, which is only concerned with the effective fuzzy competence states without listing all fuzzy competence states of fuzzy competence structures.

Step 1. Find the family of all effective fuzzy competence states of $(Q, S, \mu)$.

Step 2. Compute the knowledge states delineated by effective fuzzy competence states.

Example 5. In Example 4, the family of competencies is $\left\{\left\{\frac{0.8}{s_{4}}\right\},\left\{\frac{1}{s_{1}}, \frac{0.9}{s_{3}}\right\},\left\{\frac{0.8}{s_{1}}, \frac{0.8}{s_{2}}, \frac{0.8}{s_{3}}\right\},\left\{\frac{1}{s_{1}}, \frac{0.9}{s_{3}}, \frac{0.9}{s_{4}}\right\}\right\}$. The family of weak competencies is

$$
\left\{\varnothing,\left\{\frac{1}{s_{1}}, \frac{0.8}{s_{2}}, \frac{0.9}{s_{3}}\right\},\left\{\frac{1}{s_{1}}, \frac{0.9}{s_{3}}, \frac{0.8}{s_{4}}\right\},\left\{\frac{0.8}{s_{1}}, \frac{0.8}{s_{2}}, \frac{0.8}{s_{3}}, \frac{0.8}{s_{4}}\right\},\left\{\frac{1}{s_{1}}, \frac{0.8}{s_{2}}, \frac{0.9}{s_{3}}, \frac{0.8}{s_{4}}\right\},\left\{\frac{1}{s_{1}}, \frac{0.8}{s_{2}}, \frac{0.9}{s_{3}}, \frac{0.9}{s_{4}}\right\}\right\} .
$$


By Definition 14, the effective fuzzy competence states of $\left\{\frac{0.8}{s_{4}}\right\}$ is $\left\{\frac{0.7}{s_{2}}, \frac{0.9}{s_{3}}, \frac{0.8}{s_{4}}\right\}$. The other cases are analogous. Therefore, the family of effective fuzzy competence states is

$$
\left\{\varnothing,\left\{\frac{0.7}{s_{2}}, \frac{0.9}{s_{3}}, \frac{0.8}{s_{4}}\right\},\left\{\frac{1}{s_{1}}, \frac{0.9}{s_{3}}, \frac{0.6}{s_{4}}\right\},\left\{\frac{0.8}{s_{1}}, \frac{1}{s_{2}}, \frac{0.9}{s_{3}}, \frac{1}{s_{4}}\right\},\left\{\frac{1}{s_{1}}, \frac{0.7}{s_{2}}, \frac{0.9}{s_{3}}, \frac{1}{s_{4}}\right\},\left\{\frac{1}{s_{1}}, \frac{1}{s_{2}}, \frac{1}{s_{3}}, \frac{1}{s_{4}}\right\}\right\} .
$$

Then, $p_{\mu}(\varnothing)=\varnothing, p_{\mu}\left(\left\{\frac{0.7}{s_{2}}, \frac{0.9}{s_{3}}, \frac{0.8}{s_{4}}\right\}\right)=\left\{q_{3}\right\}, p_{\mu}\left(\left\{\frac{1}{s_{1}}, \frac{0.9}{s_{3}}, \frac{0.6}{s_{4}}\right\}\right)=\left\{q_{1}\right\}, p_{\mu}\left(\left\{\frac{0.8}{s_{1}}, \frac{1}{s_{2}}, \frac{0.9}{s_{3}}, \frac{1}{s_{4}}\right\}\right)=$ $\left.\left\{q_{2}, q_{3}\right\}, p_{\mu}\left(\left\{\frac{1}{s_{1}}, \frac{0.7}{s_{2}}, \frac{0.9}{s_{3}}, \frac{1}{s_{4}}\right\}\right)=\left\{q_{1}, q_{3}, q_{4}\right\}, p_{\mu}\left(\left\{\frac{1}{s_{1}}, \frac{1}{s_{2}}, \frac{1}{s_{3}}, \frac{1}{s_{4}}\right\}\right\}\right)=Q$. Thus, the knowledge structure induced by the competence-based fuzzy skill function is

$$
\mathcal{K}=\left\{\varnothing,\left\{q_{1}\right\},\left\{q_{3}\right\},\left\{q_{2}, q_{3}\right\},\left\{q_{1}, q_{3}, q_{4}\right\}, Q\right\},
$$

which is the same as the one in Example 4.

\section{Fuzzy interpretation functions}

For any competence structure $(S, \mathcal{C})$, Korossy $(1997,1999)$ defined a special skill multimap $\mu$ from $Q$ to $\mathcal{P}^{*}(\mathcal{C} \backslash\{\varnothing\})$. Korossy calls such a skill multimap $\mu$ an interpretation function. The concept of interpretation function can be extended to fuzzy skills and fuzzy competence structures.

Definition 15. Let $\left(S, V_{s}, \mathcal{C}\right)$ be a fuzzy competence structure. A fuzzy interpretation function is a triple $(Q, S, \mu)$, where $\mu$ is a mapping from $Q$ to $\mathcal{P}^{*}(\mathcal{C} \backslash\{\varnothing\})$ and the subsets in each $\mu(q)$ are pairwise incomparable.

Proposition 3. For a fuzzy competence space $\left(S, V_{s}, \mathcal{C}\right)$ and a fuzzy interpretation function $(Q, S, \mu)$, let $p_{\mu}: \mathcal{C} \rightarrow \mathcal{P}(Q)$ is the problem function of $(Q, S, \mu)$. If $p_{\mu}\left(T_{1} \sqcup T_{2}\right)=p_{\mu}\left(T_{1}\right) \cup p_{\mu}\left(T_{2}\right)$ for all $T_{1}, T_{2} \in \mathcal{C}$, then for all $q \in Q$, each of the competencies $C \in \mu(q)$ is an atom of $\mathcal{C}$.

Proof. Let $\mathcal{B}$ be the basis of $\left(S, V_{s}, \mathcal{C}\right)$. By Theorem 1 , assume $C \notin \mathcal{B}$ for some $C \in \mu(q)$ and $q \in Q$. Then $C=\sqcup \mathcal{B}^{\prime}$ with $\mathcal{B}^{\prime} \subseteq \mathcal{B}$. Obviously, $q \in p_{\mu}(C)=p_{\mu}\left(\sqcup \mathcal{B}^{\prime}\right)=\bigcup_{B_{i} \in \mathcal{B}^{\prime}} p_{\mu}\left(B_{i}\right)$, then $q \in p_{\mu}\left(B_{i}\right), i \in\{1,2, \cdots, n\}$. Without loss of generality, suppose $q \in p_{\mu}\left(B_{1}\right)$, then there exists $C^{\prime} \in \mu(q)$ such that $C^{\prime} \subseteq B_{1} \subset C$. That is, $C, C^{\prime} \in \mu(q)$ and $C^{\prime} \subset C$, a contradiction.

Remark 8. The reverse of Proposition 3 is unnecessarily correct even if for (dichotomous) interpretation function and (dichotomous) competence structure. Consider the following example. Let $Q=\left\{q_{1}, q_{2}, q_{3}\right\}, S=\left\{s_{1}, s_{2}, s_{3}, s_{4}, s_{5}\right\}$ and $V_{s_{i}}=\{0,1\}$ for any $i=1,2, \cdots, 5$. Consider the competence space $\mathcal{C}=\left\{\varnothing,\left\{s_{1}, s_{2}\right\},\left\{s_{2}, s_{3}\right\},\left\{s_{1}, s_{2}, s_{3}\right\},\left\{s_{3}, s_{4}, s_{5}\right\},\left\{s_{2}, s_{3}, s_{4}, s_{5}\right\}, S\right\}$. Obviously, the basis of $\mathcal{C}$ is $\left\{\left\{s_{1}, s_{2}\right\},\left\{s_{2}, s_{3}\right\},\left\{s_{3}, s_{4}, s_{5}\right\}\right\}$. An interpretation function $(Q, S, \mu)$ is defined as: $\mu\left(q_{1}\right)=\left\{\left\{s_{2}, s_{3}\right\}\right\}, \mu\left(q_{2}\right)=\left\{\left\{s_{3}, s_{4}, s_{5}\right\}\right\}, \mu\left(q_{3}\right)=\left\{\left\{s_{1}, s_{2}\right\}\right\}$, which satisfies each of the competencies $C \in \mu(q)$ is an atom of $\mathcal{C}$ for all $q \in Q$. We have $p_{\mu}\left(\left\{s_{1}, s_{2}\right\} \sqcup\left\{s_{3}, s_{4}, s_{5}\right\}\right)=Q, p_{\mu}\left(\left\{s_{1}, s_{2}\right\}\right)=$ $\left\{q_{3}\right\}, p_{\mu}\left(\left\{s_{3}, s_{4}, s_{5}\right\}\right)=\left\{q_{2}\right\}$. But, $p_{\mu}\left(\left\{s_{1}, s_{2}\right\} \cup\left\{s_{3}, s_{4}, s_{5}\right\}\right) \neq p_{\mu}\left(\left\{s_{1}, s_{2}\right\}\right) \cup p_{\mu}\left(\left\{s_{3}, s_{4}, s_{5}\right\}\right)$. Correspondingly, the knowledge structure induced by the interpretation function $(Q, S, \mu)$ is $\mathcal{K}=$ $\left\{\varnothing,\left\{q_{1}\right\},\left\{q_{2}\right\},\left\{q_{3}\right\},\left\{q_{1}, q_{2}\right\},\left\{q_{1}, q_{3}\right\}, Q\right\} . \mathcal{K}$ is not a knowledge space.

Proposition 4. For a $\sqcap$-closed fuzzy competence structure $\left(S, V_{s}, \mathcal{C}\right)$ and a fuzzy interpretation function $(Q, S, \mu)$, the following two statements are equivalent.

(a) For all $q \in Q, \mu(q)=\{C\}$ for some $C \in \mathcal{C}$;

(b) $p_{\mu}\left(T_{1} \sqcap T_{2}\right)=p_{\mu}\left(T_{1}\right) \cap p_{\mu}\left(T_{2}\right)$ for any $T_{1}, T_{2} \in \mathcal{C}$. 
Proof. $(a) \Rightarrow(b)$. For any $T_{1}, T_{2} \in \mathcal{C}$, we have $p_{\mu}\left(T_{1} \sqcap T_{2}\right) \subseteq p_{\mu}\left(T_{1}\right) \cap p_{\mu}\left(T_{2}\right)$, since the monotonicity of $p_{\mu}$. Let $q \in p_{\mu}\left(T_{1}\right) \cap p_{\mu}\left(T_{2}\right)$. We have $C \subseteq T_{1}$ and $C \subseteq T_{2}$, since $\mu(q)=\{C\}$ for some $C \in \mathcal{C}$. Thus, $C \subseteq T_{1} \sqcap T_{2}$. It follows that $q \in p_{\mu}(C) \subseteq p_{\mu}\left(T_{1} \sqcap T_{2}\right)$.

$(b) \Rightarrow(a)$. Assume $T_{1}, T_{2} \in \mu(q)$ for some $q \in Q$ and $T_{1}, T_{2} \in \mathcal{C}$. Then $q \in p_{\mu}\left(T_{1}\right) \cap p_{\mu}\left(T_{2}\right)$. By condition (2), we have $q \in p_{\mu}\left(T_{1} \sqcap T_{2}\right)$. Thus, there is $C \in \mu(q)$ such that $C \subseteq T_{1} \sqcap T_{2}$. As $T_{1} \sqcap T_{2} \subset T_{1}$ or $T_{1} \sqcap T_{2} \subset T_{2}$, then $C \subset T_{1}$ or $C \subset T_{2}$, which contradicts the incomparability condition.

Corollary 1. For a $\sqcap$-closed fuzzy competence structure $\left(S, V_{s}, \mathcal{C}\right)$ and a fuzzy interpretation function $(Q, S, \mu)$, let $(Q, \mathcal{K})$ be the knowledge structure induced by $(Q, S, \mu)$. If $(Q, S, \mu)$ satisfies Proposition $4(a)$. Then $(Q, \mathcal{K})$ is a simple closure space.

Proof. Take any $K_{1}, K_{2} \in \mathcal{K}$. Assume that $K_{i}$ is delineated by $T_{i} \in \mathcal{C}$ for any $i=1,2$. By Proposition $4, K \cap L=p_{\mu}\left(T_{1}\right) \cap p_{\mu}\left(T_{2}\right)=p_{\mu}\left(T_{1} \sqcap T_{2}\right) \in \mathcal{K}$, since $\mathcal{C}$ is $\sqcap$-closed.

\section{Conclusions}

The relationship between item set $Q$ and fuzzy set of skills $\mathcal{F}(S)$ can be established by means of a fuzzy skill function $(Q, S, \mu)$. Given that there may exist precedence relations among skills , this paper introduce the concept of fuzzy competence structure, which restricted on the possible fuzzy sets of skills. A fuzzy competence structure $\left(S, V_{s}, \mathcal{C}\right)$ is a subcollection of $\mathcal{F}(S)$ containing $S \times\{0\}$ and $S \times\{1\}$, fuzzy competence states represents individuals' levels of proficiency of skills. Theorem 1 shows that the basis of any fuzzy competence space is the collection of all the atoms.

The approach for constructing knowledge structures via fuzzy skill functions is from the perspective of latent cognitive abilities, where fuzzy skill functions are obtained by consulting experienced experts. For a fuzzy competence space $\left(S, V_{s}, \mathcal{C}\right)$, Theorem 2 shows the method for constructing the knowledge structure via a competence-based disjunctive fuzzy skill function $(Q, S, \mu)$ is only concerned with the basis of $\left(S, V_{s}, \mathcal{C}\right)$. For a $\sqcap$-closed fuzzy competence structure $\left(S, V_{s}, \mathcal{C}\right)$, Theorem 3 shows the method for constructing the simple closure space via a competence-based conjunctive fuzzy skill function $(Q, S, \mu)$ is only concerned with the minimal intersection generation group of $\left(S, V_{s}, \mathcal{C}\right)$. For a fuzzy competence structure $\left(S, V_{s}, \mathcal{C}\right)$, Theorem 4 shows the method for constructing the knowledge structure via any competence-based fuzzy skill function $(Q, S, \mu)$ just depends on effective fuzzy competence states of $(Q, S, \mu)$.

Fuzzy interpretation function is a special fuzzy skill function, which assigns to each item a nonempty family of fuzzy competence states. The algorithms we designed for delineating knowledge structures via competence-based fuzzy skill functions are also valid for fuzzy interpretation functions. For a fuzzy competence space $\left(S, V_{s}, \mathcal{C}\right)$, Proposition 3 shows that if the problem function $\left(Q, S, p_{\mu}\right)$ preserves unions in the sense that $p_{\mu}\left(T_{1} \sqcup T_{2}\right)=p_{\mu}\left(T_{1}\right) \cup p_{\mu}\left(T_{2}\right)$ for all $T_{1}, T_{1} \in \mathcal{C}$, then each competency of the fuzzy interpretation function $(Q, S, \mu)$ is an atom. However, an example in remark 8 shows that the reverse of Proposition 3 is unnecessarily correct. For a $\sqcap$-closed fuzzy competence structure $\left(S, V_{s}, \mathcal{C}\right)$, Proposition 4 shows that the problem function $\left(Q, S, p_{\mu}\right)$ preserves intersections in the sense that $p_{\mu}\left(T_{1} \sqcap T_{2}\right)=p_{\mu}\left(T_{1}\right) \cap p_{\mu}\left(T_{2}\right)$ for all $T_{1}, T_{1} \in \mathcal{C}$ if and only if for all $q \in Q$, there exists $C \in \mathcal{C}$ such that $\mu(q)=\{C\}$. The algorithms we designed in this article for delineating knowledge structures are expected to be useful for future work. 


\section{Acknowledgment}

This work is supported by the National Natural Science Foundation of China (No. 11871259, 11971287) and the Natural Science Foundation of Fujian Province (No. 2020J02043).

\section{References}

Anselmi, P., Robusto, E., \& Stefanutti, L. (2012). Uncovering the best skill multimap by constraining the error probabilities of the gain-loss model. Psychometrika, 77(4), 763-781. https://doi.org/ 10.1007/s11336-012-9286-0

Anselmi, P., Stefanutti, L., de Chiusole, D., \& Robusto, E. (2017). The assessment of knowledge and learning in competence spaces: the gain-loss model for dependent skills. British Journal of Mathematical and Statistical Psychology, 70(3), 457-479. https://doi.org/10.1111/bmsp.12095

Bartl, E., \& Belohlavek, R. (2011). Knowledge spaces with graded knowledge states. Information Sciences, 181(8), 1426-1439. https://doi.org/10.1016/j.ins.2010.11.040

Chen, J., \& de la Torre, J.(2013). A general cognitive diagnosis model for expert-defined polytomous attributes. Applied Psychological Measurement, 37, 419-437. https://doi.org/10.1177/014 6621613479818

Cosyn, E., \& Thiery, N. (2000). A practical procedure to build a knowledge structure. Journal of Mathematical Psychology, 44(3), 383-407. https://doi.org/10.1006/jmps.1998.1252

de la Torre, J., \& Douglas, J. (2008). Model evaluation and multiple strategies in cognitive diagnosis: An analysis of fraction subtraction data. Psychometrika, 73(4), 595-624. https://doi.org/10. 1007/s11336-008-9063-2

de la Torre, J. (2009). DINA model and parameter estimation: A didactic. Journal of Educational and Behavioral Statistics, 34, 115-130. https://doi.org/10.3102/1076998607309474

de la Torre, J. (2011). The generalized dina model framework. Psychometrika, 76, 179-199. https://doi.org/10.1007/s11336-011-9207-7

Doignon, J.-P., \& Falmagne, J.-C. (1985). Spaces for the assessment of knowledge. International Journal of Man-Machine Studies, 23(2), 175-196. https://doi.org/10.1016/S0020-7373(85)800316

Doignon, J.-P. (1994). Knowledge spaces and skill assignments. In G. Fischer, \& D. Laming (Eds.), Contributions to mathematical psychology, psychometrics and methodology (pp. 111-121). New York: Springer-Verlag.

Doignon, J.-P., \& Falmagne, J.-C. (1999). Knowledge spaces. Berlin, Germany: Springer-Verlag. https://doi.org/10.1007/978-3-642-58625-5

Düntsch, I., \& Gediga, G. (1995). Skills and knowledge structures. British Journal of Mathematical and Statistical Psychology, 48(1), 9-27. https://doi.org/10.1111/j.2044-8317.1995.tb01047.x

Falmagne, J.-C., Koppen, M., Villano, M., Doignon, J.-P., \& Johanessen, L. (1990). Introduction to knowledge spaces: how to build, test and search them. Psychological Review, 97(2), 204-224. https://doi.org/10.1037/0033-295X.97.2.201

Falmagne, J.-C., \& Doignon, J.-P. (2011). Learning spaces: Interdisciplinary applied mathematics. Berlin, Heidelberg: Springer. https://doi.org/10.1007/978-3-642-01039-2

Falmagne, J.-C., Albert, D., Doble, C., Eppstein, D., \& Hu, X. (2013). Knowledge spaces: Applications in education. New York, NY: Springer-Verlag. https://doi.org/10.1007/978-3-642-35329-1 
Gediga, G., \& Duntsch, I. (2002). Skill set analysis in knowledge structures. British Journal of Mathematical and Statistical Psychology, 55(2), 361-384. https://doi.org/10.1348/000711002760 554516

Heller, J. (2004). A formal framework for characterizing querying algorithms. Journal of Mathematical Psychology, 48(1), 1-8. https://doi.org/10.1016/j.jmp.2003.10.003

Heller, J., Steiner, C., Hockemeyer, C., \& Albert, D. (2006). Competence-based knowledge structures for personalised learning. International Journal on E-learning, 5(1), 75-88.

Heller, J., Ünlü, A., \& Albert, D. (2013). Skills, competencies and knowledge structures. In J.-C. Falmagne, D. Albert, C. Doble, D. Eppstein \& X. Hu (Eds.), Knowledge spaces: Applications in education (pp. 229-242). New York, NY: Springer-Verlag. https://doi.org/10.1007/978-3642-35329-1

Heller, J., Stefanutti, L., Anselmi, P., \& Robusto, E. (2015). On the link between cognitive diagnostic models and knowledge space theory. Psychometrika, 80(4), 995-1019. https://doi.org/10.1007 /s11336-015-9457-x

Heller, J., Anselmi, P., Stefanutti, L., \& Robusto, E. (2017). A necessary and sufficient condition for unique skill assessment. Journal of Mathematical Psychology, 79, 23-28. https://doi.org/10.1016 /j.jmp.2017.05.004

Heller, J. (2021). Generalizing quasi-ordinal knowledge spaces to polytomous items. Journal of Mathematical Psychology, 101, 102515. https://doi.org/10.1016/j.jmp.2021.102515

Mirsky, L. (1971). Transversal theory: An account of some aspects of combinatorial mathematics. New York: Academic Press.

Mislevy, R. J. (1996). Test theory reconceived. Journal of Educational Masurement, 33(4), 379-416. https://doi.org/10.1111/j.1745-3984.1996.tb00498.x

Kambouri, M., Koppen, M., Villano, M., \& Falmagne, J.-C. (1994). Knowledge assessment: Tapping human expertise by the QUERY routine. International Journal of Human-Computer Studies, 40(1), 119-151. https://doi.org/10.1006/ijhc.1994.1006

Karelitz, T. M. (2004). Ordered category attribute coding framework for cognitive assessments (Unpublished doctoral dissertation). University of Illinois at Urbana-Champaign.

Korossy, K. (1997). Extending the theory of knowledge spaces: A competence-performance approach. Zeitschrift fur Psychologie, 205(1), 53-82. https://doi.org/10.1300/J015v20n03-a

Korossy, K. (1999), Modeling knowledge as competence and performance, In D. Albert \& J. Lukas (Eds.), Knowledge spaces: Theories, empirical research, applications (pp. 103-132). Mahwah, NJ: Lawrence Erlbaum Associates.

Spoto, A., Stefanutti, L., \& Vidotto, G. (2016). An iterative procedure for extracting skill maps from data. Behavior Research Methods, 48, 729-741. https://doi.org/10.3758/s13428-015-06099

Stefanutti, L., \& de Chiusole, D. (2017). On the assessment of learning in competence based knowledge space theory. Journal of Mathematical Psychology, 80, 22-32. https://doi.org/10. 1016/j.jmp.2017.08.003

Stefanutti, L. (2019). On the assessment of procedural knowledge: from problem spaces to knowledge spaces. British Journal of Mathematical and Statistical Psychology, 72(2), 185-218. https://doi.org/10.1111/bmsp.12139

Stefanutti, L., Anselmi, P., de Chiusole, D., \& Spoto, A. (2020). On the polytomous generalization of knowledge space theory. Journal of Mathematical Psychology, 94, 102306. https://doi.org/10.1 016/j.jmp.2019.102306 
Sun, W., Li, J., Ge, X., \& Lin, Y. (2021). Knowledge structures delineated by fuzzy skill maps. Fuzzy Sets and Systems, 407, 50-66. https://doi.org/10.1016/j.fss.2020.10.004

von Davier, M. (2008). A general diagnostic model applied to language testing data. British Journal of Mathematical and Statistical Psychology, 61, 287-307. https://doi.org/10.1002/j.23338504.2005.tb01993.x

Zadeh, L. A. (1965). Fuzzy sets. Information and control, 8(3), 338-353. https://doi.org/10.1016/S 0019-9958(65)90241-X

Zhan, P., Jiao, H., \& Liao, D. (2017). Cognitive diagnosis modelling incorporating item response times. British Journal of Mathematical and Statistical Psychology, 71, 262-286. https://doi.org/ 10.1111/bmsp.12114 